\title{
DIFFERENTIAL RECEPTOR-FUNCTIONALITY OF THE TWO DISTINCT RECEPTOR PROTEINS FOR MOUSE HEPATITIS VIRUS
}

\author{
N. Ohtsuka, ${ }^{1}$ Y. K. Yamada, ${ }^{2}$ K. Saeki, ${ }^{1}$ and F. Taguchi' \\ 'National Institute of Neuroscience, NCNP \\ 4-1-1 Ogawahigashi, Kodaira \\ Tokyo 1871, Japan \\ ${ }^{2}$ National Institute of Infectious Diseases \\ 4-7-1 Gakuen, Musashimurayama \\ Tokyo 190-122, Japan
}

\section{ABSTRACT}

We compared the virus-binding activity and receptor-functionality of the receptor proteins isolated from mouse hepatitis virus (MHV)-susceptible BALB/c mice (MHVR1) and MHV-resistant SJL mice (MHVR2). By using a soluble receptor protein which lacked the transmembrane and intracytoplasmic domains, virus overlay protein blot assay and neutralization tests showed that MHVR1 bound to JHM cl-2 virus with 300-500 times higher efficiency than to MHVR2. MHVR 1 was revealed to have 10-30 fold higher receptor-functionality than MHVR2 when examined by measuring virus-binding to the receptor expressed on the cell surface. These findings suggested that the differences in susceptibility between BALB/c and SJL mice may depend upon the genotype of the MHV receptor.

\section{INTRODUCTION}

The receptor proteins for mouse hepatitis virus (MHV) isolated from MHV-susceptible $\mathrm{BALB} / \mathrm{c}$ mice was reported to interact with the virus particles in virus overlay protein blot assay (VOPBA), although such proteins were not detected in SJL mice resistant to MHV (Boyle et al., 1987). This receptor was thereafter identified as Bgp $1^{a}$ with four domains (the splice variant of Bgp $1^{\text {a }}$ containing two domains is designated as MHVR1 in this paper) which is classified as a carcinoembryonic antigen gene family member in the immunoglobulin super family and is a functional receptor protein for MHV-A59 (Dveksler et al., 1991). This finding could imply that the difference in susceptibility between BALB/c and 
SJL mouse strains to MHV infection may be due to the presence or absence of the functional receptor. In SJL mice, however, the homologous protein to MHVR1, named Bgp $1^{\text {b }}$ with two domains (called as MHVR2 in this paper), was found to serve as a functional receptor as well (Yokomori and Lai, 1992a, b), These results imply that the difference in susceptibility to MHV could not be simply explained by the presence or absence of functional receptors. To understand how the resistance of mice to MHV is controlled, we have compared the virus-binding activity and receptor-functionality of these proteins.

\section{RESULTS AND DISCUSSION}

To examine the virus-binding activity, we utilized soluble forms of the receptor proteins which lacked the transmembrane domain and intracytoplasmic tail and were tagged with influenza HA epitope. The genes for these soluble receptor proteins were prepared by PCR as described previously (Yamada et al., 1993) (Fig. 1). These genes were transfected into RK13 cells by electroporation and soluble receptors were expressed with the vaccinia virus T7 system (Fuerst et al., 1986). Soluble forms of MHVR1 and MHVR2 secreted in the culture fluid were concentrated by ultrafiltration (millopore) and used to compare the virus-binding activity by VOPBA (Kubo et al., 1994; Taguchi, 1995). As has been reported (Boyle et al., 1987), we have observed that JHM cl-2 virus (Taguchi et al., 1985) bound to MHVR1 but not to MHVR2 (data not shown). Furthermore we performed a similar experiment by dot-blot hybridization to analyze the virus-binding activity quantitatively. The result was that MHVR1 had 300 times higher virus-binding activity than did MHVR2 (data not shown). Neutralization tests of JHM with the soluble form of the receptor proteins revealed that MHVR1 has a 500-fold higher neutralizing activity than MHVR2 (Fig. 2). These findings by VOPBA and neutralization assay indicate that there is a marked difference between MHVR1 and MHVR2 in virus-binding activity.
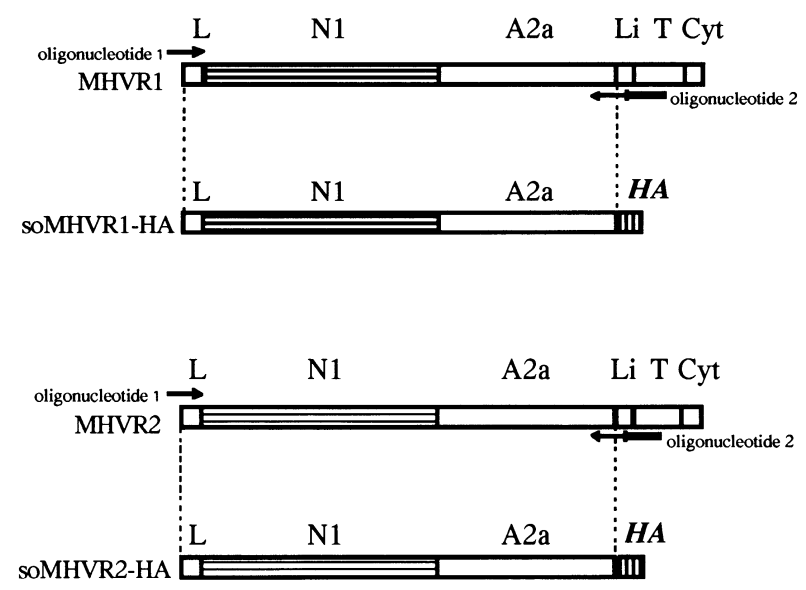

Figure 1. Structure of the genes used to express soluble MHVR1 and MHVR2. MHVR1 and MHVR2 are composed of two major domains, $\mathrm{N}$ and $\mathrm{A} 2$. L, Li, T, and Cyt indicate leader sequence, linker region, transmembrane domain and intracytoplasmic domain, respectively. The genes were designed to delete the transmembrane and intracytoplasmic domains and were tagged with HA epitope by PCR. 
Figure 2. Neutralization of JHM by soluble MHVR1 and MHVR2. Soluble MHVR1 and MHVR2 as well as control culture fluids diluted two fold were mixed with JHM and incubated at RT for $1 \mathrm{~h}$. The virus titres in the mixtures were plaque assayed on DBT cells. The rate of inhibition of virus infectivity is shown as neutralizing efficiency.
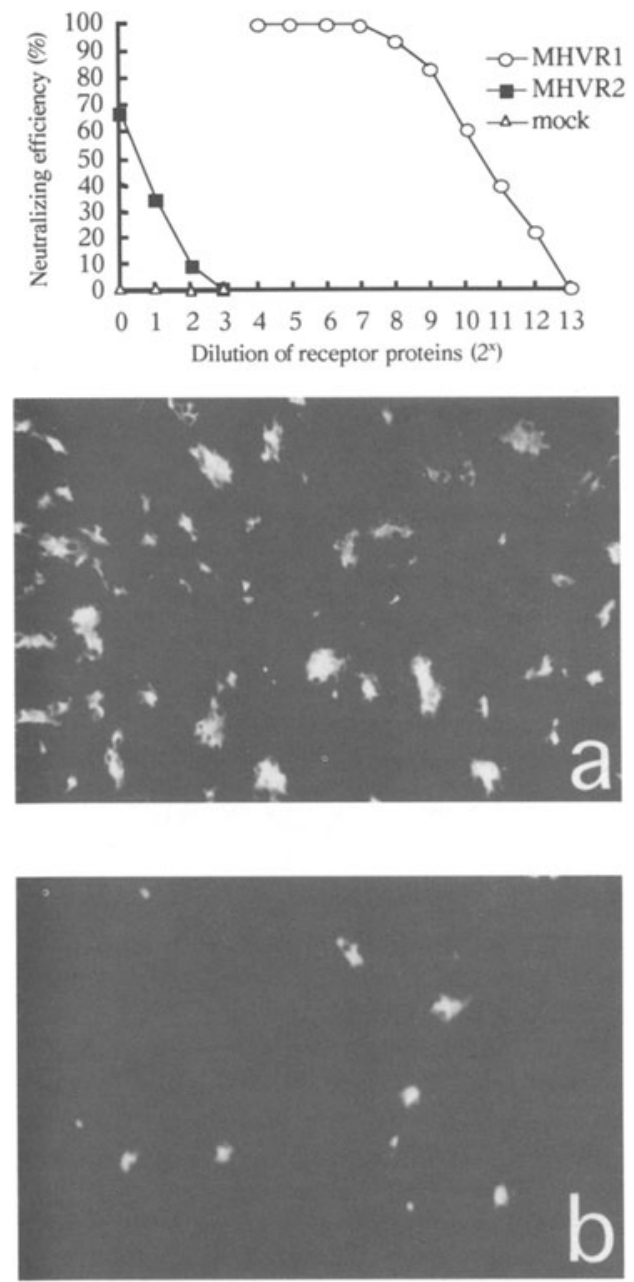

Figure 3. Immunofluorescence of BHKMHVR1 and BHK-MHVR2 cells infected with JHM. BHK-MHVR1 (a), BHK-MHVR2 (b) and control BHK (c) cells infected with JHM at an m.o.i. of 0.5 were fixed with acetone at $10 \mathrm{~h}$ post-inoculation and examined for the presence of JHM antigen with a JHM-specific MAb.

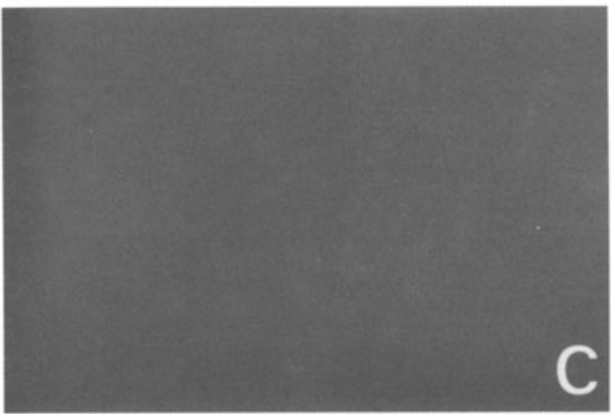


We also compared the receptor-functionality of these proteins using MHVR expressed on cell membranes. MHVR1 and MHVR2 with transmembrane and intracytoplasmic domains were expressed in BHK-21 cells as described previously (Suzuki and Taguchi, 1996). There was a significant difference in the susceptibility to JHM between BHK cells expressing MHVR1 (BHK-MHVR1) and BHK cells expressing MHVR2 (BHK-MHVR2) (Fig.3). The numbers of fused BHK-MHVR1 cells were 10-fold more than that BHK-MHVR2 after infection at a multiplicity of infection (m.o.i.) of 0.5 (Fig. 3), and 30-fold higher at a m.o.i. of 0.005 . We also investigated virus growth in these cells. JHM grew more efficiently in the BHK-MHVR1 cells than BHK-MHVR2. The difference was 6-60 times (data not shown). These results indicate that MHVR1 served as a highly efficient receptor for JHM, 10-30 fold higher as compared with MHVR2.

In the present study, a quantitative difference was shown in virus-binding ability between MHVR1 and MHVR2. Such a difference could account for the difference in susceptibility of cells to JHM infection. The finding that BHK-MHVR1 was 10-30 fold more susceptibility than BHK-MHVR2 cells could indicate the possibility that the resistance of SJL mice to JHM could be accounted for by the low virus-affinity of MHVR2 as compared with the MHVR1 of the susceptible BALB/c mouse strain. A 10 to 30-fold difference in virus affinity would be amplified into a huge difference in virus growth after repeated cycles of infection within a few days after the initial infection, which could result in the fatal disease of susceptible BALB/c mice and the survival of SJL mice. To investigate this possibility, susceptibilities of BALB/c mice whose MHVR1 gene has been replaced by the MHVR2 gene and SJL mice with the MHVR1 gene should be studied.

\section{REFERENCES}

Boyle J.F. , Weismiller D.G., and Holmes K.V., 1987, Genetic resistance to mouse hepatitis virus correlates with absence of virus-binding activity on target tissues, J. Virol. 61: 185-189.

Dveksler G.S., Pensiero M.N., Cardellichio C.B., Williams R.K., Jiang G., Holmes K.V. and Diffenbach, C.W., 1991, Cloning of the mouse hepatitis virus (MHV) receptor: expression in human and hamster cell lines confers susceptibility to MHV, J. Virol. 65: 6881-6891.

Fuerst, T.R., Niles, E.G., Studier, F.W., and Moss, B., 1986, Eukaryotic transient expression system based on recombinant vaccinia virus that synthesizes T7 RNA polymerase, Proc. Natl. Acad. Sci. USA 83: 8122-8126.

Kubo, H., Yamada, Y.K. and Taguchi, F., 1994, Localization of neutralizing epitopes and the receptor-binding site within the amino-ternimal 330 amino acids of the murine coronavirus spike protein, J. Virol. 68 : 5403-5410.

Suzuki, H., and Taguchi, F., 1996, Analysis of receptor-binding site of murine coronavirus spike protein, J. Virol. 70: $2632-2636$.

Taguchi, F., Siddell, S.G., Wege, H. and ter Meulen, V., 1985, Characterization of a variant virus selected in rat brains after infection by coronavirus mouse hepatitis virus JHM, J. Virol. 54: 429-435.

Yamada, Y.K., Abe, M., Yamada, A. and Taguchi, F., 1993, Detection of mouse hepatitis virus by the polymerase chain reaction and its application to the rapid diagnosis of infection, Lab. Anim. Sci. 43: 285-290.

Yokomori K. and Lai. M.M.C., 1992a, Mouse hepatitis virus utilizes two carcionoembryonic antigens as alternative receptors, J. Virol. 66: 6194-6199.

Yokomori, K., and Lai. M.M.C., 1992b, The receptor for mouse hepatitis virus in the resistant mouse strain SJL is functional: Implications for the requirement of a second factor for viral infection, J. Virol. 66: 6931-6938. 